\title{
El papel de la IEP y del Optimismo/Pesimismo disposicional en la resolución de problemas sociales: Un estudio con alumnos de trabajo social
}

\section{J.M. Augusto Landa, M.C. Aguilar-Luzón, M. F. Salguero de Ugarte}

Departamento de Psicología,

Facultad de Humanidades y Ciencias de la Educación, Universidad de Jaén

\section{España}




\section{Resumen}

Introducción. El presente estudio analiza la validez predictiva de la inteligencia emocional percibida y del optimismo/pesimismo disposicional en la resolución de problemas sociales en estudiantes de trabajo social.

Método. Un total de 122 estudiantes de segundo curso de la Diplomatura de Trabajo Social de la Universidad de Jaén (España) completaron las siguientes escalas en su versión castellana: TMMS, LOT-R y SPSI-R.

Resultados. Los análisis de correlaciones han mostrado relaciones significativas entre las escalas del TMMS y LOT-R con las sub-escalas del SPSI-R. Los análisis de regresión jerárquica han encontrado que el factor de IEP denominado Claridad emocional predice parte de la varianza de las sub-escalas del SPSI-R denominadas Orientación positiva hacia los problemas, Resolución racional de problemas y Estilo de evitación. Por otra parte, el factor Atención a las emociones explicó parte de la varianza de la sub-escala Orientación negativa hacia el problema. Así mismo el LOT-R predice parte de la varianza de las sub-escalas del SPSI-R.

Discusión y Conclusión. Estos resultados apuntan que los futuros trabajadores sociales con alta claridad emocional y que muestran actitudes optimistas, están en mejores condiciones de afrontar y solucionar problemas sociales.

Palabras Clave: Inteligencia Emocional; Resolución de Problemas Sociales; Optimismo; Trabajo Social

Recibido: 02/04/08 Aceptación Provisional: 20/04/08 Aceptación Definitiva: 20/05/08 


\begin{abstract}
Introduction. The present study examines the predictive validity of perceived emotional intelligence and an optimism / pessimism disposition in solving social problems in social work students.

Method. The TMMS, LOT-R and SPSI-R questionnaires were used in this study. The sample comprised 122 students from the second year of the Bachelor's degree program in Social Work at the University of Jaén (Spain).

Results. Analysis of correlations showed significant relationships between the TMMS and LOT-R scales and the SPSI-R sub-scales. Hierarchical regression analyses revealed that the IEP factor called emotional clarity predicts part of the variance of the SPSI-R sub-scales, those referring to Positive Problem Orientation, Rational Problem Solving and Avoidance Style. Elsewhere, the emotional attention factor explained part of the variance of the Negative Problem Orientation sub-scale. Likewise, the LOT-R predicts part of the variance of the SPSI-R sub-scales.
\end{abstract}

Discussion and Conclusion. These results suggest that future social workers with high emotional clarity and optimistic attitude are better able to tackle and resolve social problems.

Keywords: Emotional intelligence; social problem solving; optimism; social work.

Received: 04/02/08Ｉnitial Acceptance: 04/20/08ＩFinal Acceptance: 05/20/08 


\section{Introducción}

Considerando que la profesión de Trabajo Social "promueve la resolución de problemas en las relaciones humanas, el cambio social, el poder de las personas mediante el ejercicio de sus derechos y su liberación y la mejora de la sociedad. Mediante la utilización de teorías sobre el comportamiento humano y los sistemas sociales, el trabajo social interviene en los puntos en los que las personas interactúan con su entorno" [Definición de la Federación Internacional de Trabajadores Sociales (FITS), en su Asamblea General celebrada en Montreal en Julio del 2000], podríamos deducir la importancia que, para los estudiantes de Trabajo Social, tendría la generación de estrategias que les permitan afrontar con garantías su futura profesión. Las habilidades que posee una persona para resolver problemas de tipo interpersonal, ocupan un papel central en el estudio de la Psicología. Además, siguiendo a Esquivias, González y Muria (2003), ser capaz de resolver problemas habilita a la persona para adaptarse a las demandas del medio ambiente, y le permite modificarlas de manera parcial. En este sentido y según el modelo postulado por D'Zurilla y Nezu (1999), las habilidades de resolución de problemas sociales comprenden procesos cognitivos, afectivos y conductuales a través de los cuales los sujetos tratan de identificar, descubrir o adaptar respuestas hacia situaciones que se producen en la vida cotidiana. Según D’Zurilla, Nezu y Maydeu-Olivares (2004), la capacidad de resolución de problemas consiste en dos componentes principales, parcialmente independientes: a) orientación al problema (componente motivador) y b) habilidades específicas de solución de problemas (habilidades cognoscitivas y del comportamiento por las que una persona procura entender el problema y encontrar soluciones eficaces o maneras de hacer frente a los problemas). Concretamente, en el modelo presentado por los citados autores, se identifican cuatro habilidades importantes:

a) definición y formulación del problema;

b) generación de soluciones alternativas;

c) toma de decisión;

d) puesta en práctica y verificación de la solución.

A partir de este modelo, D'Zurilla y Nezu (1990) desarrollaron el Inventario de solución de problemas sociales (SPSI). Posteriormente, y teniendo en cuenta los resultados hallados en diferentes estudios empíricos (Maydeu-Olivares y D'Zurilla, 1996; D'Zurilla, Nezu y Maydeu-Olivares, 1998), desarrollaron el inventario de solución de problemas denominado (SPSI-R). Existen varios estudios que han relacionado las habilidades de 
solución de problemas sociales con otras variables criterio tales como estilos de afrontamiento (Kant, D’Zurilla y Maydeu-Olivares, 1997), el riesgo de suicidio en adolescentes (Chang, 1998), el padecimiento de trastornos psicológicos (Nezu y D'Zurilla, 1989), los estilos de toma de decisión (Morera, Madey-Olivares, Nygren, White, Fernández y Skewes, 2006), o la exploración de diferencias en cuanto al sexo y la edad (D'Zurilla, Maydeur-Olivares y Kant, 1998).

Uno de los temas que recientemente está recibiendo un mayor empuje científico es el optimismo disposicional. Se entiende por optimismo la tendencia a creer que, en el futuro ocurrirán resultados favorables o éxitos, mientras que su contrario, el pesimismo, hace alusión a la creencia de que en el futuro ocurrirán resultados desfavorables o negativos (Avia y Vázquez, 1999; Carver y Scheier, 2001; Chang, 2001; Sheier, Carver y Bridges, 2001). Por tanto, el optimismo incluye expectativas de control sobre los propios resultados positivos en un futuro así como un cierto componente de eficacia personal (Gillham, Shatte, Reivich y Seligman, 2001). Varios estudios han constatado la relación entre el optimismo/pesimismo disposicional y determinadas variables psicológicas y de salud. Así, se ha comprobado que el pesimismo disposicional está relacionado con una mayor sensación o experimentación de síntomas físicos negativos (Chang, 1998; Maydeu-Olivares y D’Zurilla, 1996), mientras que, aquellas personas que puntúan alto en optimismo disposicional informan de una menor frecuencia e intensidad de síntomas físicos negativos (Remor, Amorós y Carboles, 2006). Con respecto a la relación entre optimismo disposicional y estrategias de afrontamiento al estrés, varios estudios han constatado que, los sujetos optimistas afrontan con mayor posibilidad de éxito las situaciones estresantes, utilizando estrategias de afrontamiento activo, es decir, aquellas estrategias que tratan de incidir directamente sobre la fuente de estrés (Chico, 2002; Martínez-Correa, Reyes del Paso, García-León y González-Jareño, 2006). Sin embargo, son pocos los trabajos que han analizado la relación entre el optimismo/pesimismo disposicional y su influencia en la resolución de problemas sociales. En esta línea, los resultados obtenidos por Vera-Villarroel y Guerrero (2003), indican que las personas pesimistas se diferencian de las optimistas, en cuanto a la orientación positiva hacia los problemas, puesto que, las personas caracterizadas por ser optimistas, muestran una solución más racional de los problemas. No obstante, creemos necesaria la puesta en marcha de un mayor número de investigaciones dirigidas a esclarecer la relación que se establece entre la disposición optimista/pesimista que mostramos las personas ante determinados problemas sociales. 
En este sentido, la inteligencia emocional se ha mostrado como un importante factor, a la hora de predecir eventos vitales significativos y emotivos. De ahí que en este trabajo, de acuerdo con autores como Salovey y Mayer (1990) y Mayer y Salovey (1997), partimos de la consideración de que la inteligencia emocional hace referencia a la habilidad para reconocer los significados de las emociones, utilizándolas para razonar y resolver problemas, adoptando así un carácter cognitivo. Según estos autores, la inteligencia emocional hace referencia a:

1) habilidad para identificar y percibir las emociones propias y las de los demás.

2) destreza para regular y modificar nuestro estado de ánimo de forma adecuada.

3) habilidad para mejorar el pensamiento.

Uno de los auto-informes más utilizados en la medición de la inteligencia emocional, es el Trait Meta-Mood Scale (TMMS) desarrollado por Salovey, Mayer, Goldman, Turvery y Palfai (1995). Este instrumento se encuadra dentro del término conocido por los investigadores como Inteligencia Emocional Percibida (IEP), que hace referencia al conocimiento que tienen los individuos de sus propias habilidades emocionales y que no siempre coincide con su capacidad real (Extremera y Fernández-Berrocal, 2005a; Salovey, Woolery, Stroud y Epel, 2002). Recientes estudios, indican que la IEP se muestra como un importante predictor de síntomas somáticos y de la calidad de vida (Thompson, Walz, Croyle y Pepper, 2007). Además, otros resultados sugieren asociaciones significativas entre IEP y ajuste psicológico, controlando factores de personalidad y estados emocionales (Augusto, López-Zafra, Martínez de Antoñana y Pulido, 2006; Extremera, Fernández-Berrocal, 2005a) o incluso, se relaciona con el sentimiento de satisfacción general (Berrios, Augusto-Landa y Aguilar-Luzón, 2006).

El papel de la IEP y el optimismo/pesimismo en la predicción del ajuste psicológico (percepción de estrés y satisfacción de vida), también ha sido abordado por autores como Extremera, Duran y Rey (2007), con una muestra de adolescentes. Sus resultados muestran que los participantes que obtienen altas puntuaciones en las sub-escalas del TMMS, Claridad y Regulación emocional, son quienes muestran mayor satisfacción de vida y menor percepción de estrés, efecto que, en parte, puede ser considerado independiente de sus disposiciones optimistas y pesimistas. En esta línea, el presente estudio pretende examinar las relaciones entre las dimensiones del TMMS, el optimismo/pesimismo disposicional y la resolución de problemas sociales en una muestra de estudiantes de trabajo social. Concretamente, examinaremos la validez incremental de las dimensiones del TMMS 
(Atención, Claridad y Regulación) sobre las dimensiones del SPSI-R (orientación positiva hacia el problema, orientación negativa hacia el problema, solución racional de problemas, impulsividad y estilo de evitación) considerando los efectos del optimismo/pesimismo disposicional. Para ello, las hipótesis formuladas son las siguientes:

1.- Esperamos encontrar relaciones significativas entre los componentes del TMMS, LOT-R y SPSI-R.

2.- Esperamos que, los componentes del TMMS y LOT-R, actúan como buenos predictores de las distintas sub-escalas recogidas en el SPSI-R.

3.- Esperamos que existan diferencias significativas en cuanto al sexo en las dimensiones del TMMS, LOT-R y SPSI-R.

\section{Método}

\section{Participantes}

Los participantes en este estudio han sido 122 (23 hombres y 99 mujeres) estudiantes de segundo curso de la Diplomatura en Trabajo Social de la Universidad de Jaén. La edad de los participantes oscilaba entre los 18 y 28 años (Media = 20,71; D.T. =2,09).

\section{Materiales}

Inventario de Solución de Problemas Sociales - Revisado (SPSI-R; D'Zurilla, Nezu y Maydeu-Olivares, 1998). Se compone de 52 ítems con una escala de respuesta tipo Likert con un rango de puntuación de 0 a 4 y repartidos en cinco dimensiones:

a) Orientación positiva hacia los problemas (OPP) (5 ítems): ha sido descrita como una dimensión cognitiva que consiste en tomar los problemas como un desafío; recoge la creencia de la persona sobre su capacidad para poder resolverlos (auto-eficacia).

b) Orientación negativa hacia el problema (ONP) (10 ítems): constituye una respuesta cognitivo-emocional que implica una tendencia a ver los problemas como una amenaza imposible de resolver, de manera que, recoge la creencia de la persona sobre su poca capacidad para resolverlos.

c) Resolución racional de problemas (RRP) (20 ítems): es una dimensión adaptativa que consiste en la aplicación racional y sistemática de los principios y técnicas de resolución de problemas. Esta escala puede descomponerse en cuatro sub-escalas de 5 ítems cada una: 1) 
definición y formulación del problema, 2) generación de soluciones alternativas, 3) toma de decisión, y 4) ejecución de la solución y verificación.

d) Estilo impulsivo/irreflexión (EII) (10 ítems): hace referencia a un perfil inadecuado de resolución de problemas, la persona intenta resolver problema de forma precipitada e incompleta.

e) Estilo de evitación (EE) (7 ítems): se caracteriza por aplazar la solución de los problemas; son sujetos pasivos y dependientes.

También puede calcularse una puntuación total del inventario sumando las medias obtenidas en las cinco dimensiones. En la presente investigación, hemos utilizado la versión española adaptada a estudiantes universitarios por Calero, Luna, Vera-Villarroel y GonzálezTrujillo (2001). En sus estudios de validez con muestra de universitarios españoles, estos autores, encontraron que la orientación negativa hacia el problema explicaba un $26.511 \%$ de la varianza total; estilo de evitación explica un $10.459 \%$ de la varianza total; el factor impulsividad explica un $4.729 \%$ de la varianza total; solución racional de problemas explica el $29.004 \%$ de la varianza total. La estructura factorial encontrada es similar a la de la versión en inglés del SPSI, propuesto por Maydeu-Olivares, D’Zurilla y Kant (1994). La dimensión o factor que mejor correlaciona con medidas de ansiedad o de malestar es la de orientación negativa hacia los problemas (ONP).

Test de orientación vital-Revisado (LOT-R; Scheier, Carver y Bridges, 1994). Se compone de 10 ítems ( 3 positivos, 3 negativos y 4 de relleno) valorados en una escala con formato de respuesta tipo Likert con un rango de puntuación que oscila entre 1 (totalmente en desacuerdo) hasta 5 (totalmente de acuerdo). Esta escala mide el optimismo vs. pesimismo disposicional. La versión española fue desarrollada por Otero, Luengo, Romero, Gómez y Castro (1998), si bien nosotros hemos utilizado la versión validada al castellano por Ferrando, Chico y Tous (2002) que presenta un alpha de Cronbach de 0.68 en una muestra de estudiantes universitarios.

Trait Meta-Mood Scale (TMMS; Salovey, Mayer, Goldman, Turvey y Palfai, 1995). Este instrumento evalúa la Inteligencia Emocional Percibida (IEP), es decir, el metaconocimiento que las personas tienen de sus habilidades emocionales. Se compone de una escala formada por 24 ítems tipo Likert con un rango de puntuación que oscila entre 1 y 5 . La escala está compuesta por 3 dimensiones de tipo interpersonal formadas por 8 ítems cada una: 
Atención a los sentimientos (por ejemplo, "pienso en mi estado de ánimo constantemente"), Claridad emocional (por ejemplo, "frecuentemente me equivoco con mis sentimientos") y Reparación emocional (por ejemplo, "aunque a veces me siento triste, suelo tener una visión optimista"). En este estudio, se ha utilizado la versión adaptada al castellano que presenta un Alfa de Cronbach de 0.86 para el componente Atención a los sentimientos, $\alpha=0.90$ para el componente Claridad de sentimientos o claridad emocional y un $\alpha=0.86$ para el componente Reparación emocional (Fernández-Berrocal, Extremera y Ramos, 2004). Los coeficientes de consistencia interna $(\alpha)$, así como las medias y desviaciones típicas obtenidos en la presente investigación con cada una de las medidas consideradas pueden verse en la Tabla 1.

Tabla 1.- Medias, desviaciones típicas y Alpha de Cronbach de las diferentes variables.

\begin{tabular}{l|c|c|c}
\hline Medidas & Media & D.T & $\boldsymbol{\alpha}$ \\
\hline TMMS - Atención & 27.40 & 5.39 & 0.85 \\
\hline TMMS - Claridad & 25.61 & 4.66 & 0.74 \\
\hline TMMS - Regulación & 26.06 & 6.20 & 0.85 \\
\hline LOT-R-Optimismo & 10.80 & 2.21 & 0.62 \\
\hline LOT-R- Pesimismo & 9.25 & 2.22 & 0.48 \\
\hline SPSI-R- OPP & 12.58 & 3.75 & 0.66 \\
\hline SPSI-R- ONP & 18.55 & 7.64 & 0.86 \\
\hline SPSI-R- RRP & 46.80 & 13.35 & 0.91 \\
\hline SPSI-R- EII & 12.18 & 7.32 & 0.86 \\
\hline SPSI-R- EE & 8.30 & 4.98 & 0.77 \\
\hline
\end{tabular}

Nota: OPP: Orientación positiva hacia los problemas; ONP: Orientación negativa hacia los problemas; RRP: Resolución racional de problemas; EII: Estilo de impulsividad/irreflexión; EE: Estilo de evitación.

\section{Resultados}

En primer lugar, para analizar las relaciones que se establecen entre cada una de las dimensiones consideradas en el presenta trabajo, se llevaron a cabo análisis de correlación de Pearson entre las dimensiones de la IEP, los componentes del SPSI-R y el optimismo/pesimismo. Los resultados pueden observarse en la Tabla 2.

Tabla 2.- Correlaciones entre las diferentes medidas

\begin{tabular}{lllllllllll}
\hline & 1 & 2 & 3 & 4 & 5 & 6 & 7 & 8 & 9 & 10 \\
\hline 1. Atención & 1 & & & & & & & & & \\
2. Claridad & 0.16 & 1 & & & & & & & \\
3. Regulación & -0.11 & $0.28^{* *}$ & 1 & & & & & & \\
4. Optimismo & $-0.18^{*}$ & 0.09 & $0.53^{* *}$ & 1 & & & & & \\
5. Pesimismo & $-0.18^{*}$ & 0.02 & $-0.28^{* *}$ & $0.43^{* *}$ & 1 & & & & \\
6. OPP & 0.00 & $0.41^{* *}$ & $0.35^{* *}$ & $0.38^{* *}$ & $0.19^{*}$ & 1 & & & & \\
\hline
\end{tabular}




\begin{tabular}{|c|c|c|c|c|c|c|c|c|c|c|}
\hline 7. ONP & $0.39 * *$ & -0.06 & $-0.28 * *$ & $-0.40 * *$ & $-0.39 * *$ & $-0.36 * *$ & 1 & & & \\
\hline 8. RRP & 0.18 & $0.29 * *$ & 0.14 & 0.08 & 0.08 & $0.56 * *$ & 0.08 & 1 & & \\
\hline 9. EII & 0.00 & -0.11 & 0.05 & 0.00 & $-0.26 * *$ & -0.09 & $0.34 * *$ & $0.29 * *$ & 1 & \\
\hline 10.EE & 0.11 & -0.14 & 0.00 & -0.04 & $-0.23 * *$ & $-0.25 * *$ & $0.57 * *$ & 0.01 & $0.49 * *$ & 1 \\
\hline
\end{tabular}

La dimensión de IEP, atención a los sentimientos se relacionó de manera positiva con la sub-escala del SPSI-R, orientación negativa hacia los problemas y, negativamente con las sub-escalas del LOT-R, optimismo y pesimismo disposicional. La dimensión de IEP claridad de sentimientos, correlacionó de forma positiva con regulación emocional y con las subescalas del SPSI-R, orientación positiva hacia los problemas y resolución racional de problemas. Por otra parte, la dimensión de IEP regulación emocional, se relacionó de manera positiva con los componentes del LOT-R, optimismo y pesimismo y con la sub-escala del SPSI-R, orientación positiva hacia los problemas, si bien, se establecen relaciones negativas con la sub-escala del SPSI-R, orientación negativa hacia los problemas. La escala del LOT-R optimismo disposicional correlacionó a nivel positivo con las sub-escala del SPSI-R, orientación positiva hacia los problemas, y a nivel negativo con orientación negativa hacia los problemas. La escala del LOT-R pesimismo disposicional, correlacionó a nivel positivo con la sub-escala del SPSI-R, orientación positiva hacia los problemas y, a nivel negativo, con orientación negativa hacia los problemas, estilo de impulsividad/irreflexión y estilo de evitación.

Seguidamente, con el objetivo de comprobar el aporte de los componentes de la IEP y del optimismo/pesimismo disposicional en la resolución de problemas sociales, se llevaron a cabo análisis de regresión jerárquicos por pasos (ver tabla 3). Las variables dependientes fueron las 5 sub-escalas del inventario SPSI-R: orientación positiva hacia los problemas, orientación negativa hacia los problemas, resolución racional de problemas, estilo de impulsividad/irreflexión y estilo de evitación. Cada variable dependiente se introdujo en el modelo de forma individual.

Tabla 3.- Análisis de Regresión por pasos

\begin{tabular}{llllll}
\hline & $\mathrm{R}^{2}$ & $\mathrm{~F}$ & $\beta$ & $\mathrm{P}$ & $\Delta \mathrm{R}^{2}$ \\
\hline (Criterio: Orientación positiva hacia los problemas) & & & & & \\
Paso 1: Covariable & 0.02 & 1.04 & & & 0.00 \\
1. Sexo & & & 0.03 & 0.72 & \\
2. Edad & & & -0.12 & 0.18 & \\
\hline
\end{tabular}




\begin{tabular}{|c|c|c|c|c|c|}
\hline Paso 2: Subescalas del LOT-R & 0.15 & 5.20 & & & $0.12 * *$ \\
\hline Optimismo & & & 0.36 & $0.00 * *$ & \\
\hline Pesimismo & & & 0.01 & 0.85 & \\
\hline Paso 3: TMMS Subscales & 0.30 & 6.96 & & & $0.18^{* *}$ \\
\hline Atención & & & 0.02 & 0.79 & \\
\hline Claridad & & & 0.35 & $0.00 * *$ & \\
\hline Regulación & & & 0.08 & 0.39 & \\
\hline (Criterio: Orientación negativa hacia los problemas) & & & & & \\
\hline Paso 1: Covariable & 0.11 & 6.99 & & & $0.09 * *$ \\
\hline 1. Sexo & & & -0.21 & $0.01 * *$ & \\
\hline 2. Edad & & & 0.22 & $0.01 * *$ & \\
\hline Paso 2: Subescalas del LOT-R & 0.27 & 10.65 & & & $0.24 * *$ \\
\hline Optimismo & & & -0.28 & $0.00^{* *}$ & \\
\hline Pesimismo & & & -0.20 & $0.03 *$ & \\
\hline Paso 3: TMMS Subscales & 0.36 & 8.97 & & & $0.12 * *$ \\
\hline Atención & & & 0.30 & $0.00^{* *}$ & \\
\hline Claridad & & & -0.07 & 0.37 & \\
\hline Regulación & & & -0.03 & 0.77 & \\
\hline (Criterio: Resolución racional de problemas) & & & & & \\
\hline Paso 1: Covariable & 0.02 & 1.14 & & & 0.00 \\
\hline 1. Sexo & & & -0.03 & 0.73 & \\
\hline 2. Age & & & -0.14 & 0.14 & \\
\hline Paso 2: Subescalas del LOT-R & 0.03 & 0.76 & & & 0.03 \\
\hline Optimismo & & & 0.05 & 0.66 & \\
\hline Pesimismo & & & 0.05 & 0.62 & \\
\hline Paso 3: TMMS Subscales & 0.13 & 2.45 & & & $0.10^{* *}$ \\
\hline Atención & & & 0.17 & 0.06 & \\
\hline Claridad & & & 0.24 & $0.01 * *$ & \\
\hline Regulación & & & 0.04 & 0.73 & \\
\hline (Criterio: Estilo de impulsividad/irreflexión) & & & & & \\
\hline Paso 1: Covariable & 0.01 & 0.46 & & & 0.01 \\
\hline 1. Sex & & & -0.01 & 0.88 & \\
\hline 2. Age & & & 0.09 & 0.36 & \\
\hline Paso 2: Subescalas del LOT-R & 0.09 & 2.96 & & & $0.06^{* *}$ \\
\hline Optimismo & & & 0.16 & 0.11 & \\
\hline Pesimismo & & & -0.34 & $0.00^{* *}$ & \\
\hline Paso 3: TMMS Subscales & 0.12 & 2.20 & & & 0.06 \\
\hline Atención & & & -0.01 & 0.90 & \\
\hline Claridad & & & -0.15 & 0.12 & \\
\hline Regulación & & & 0.14 & 0.19 & \\
\hline (Criterio: Estilo de evitación) & & & & & \\
\hline Paso 1: Covariable & 0.01 & 0.72 & & & 0.01 \\
\hline 1. Sex & & & -0.11 & 0.23 & \\
\hline 2. Age & & & -0.02 & 0.81 & \\
\hline Paso 2: Subescalas del LOT-R & 0.06 & 1.94 & & & $0.03 * *$ \\
\hline Optimismo & & & 0.07 & 0.47 & \\
\hline Pesimismo & & & -0.26 & $0.01 * *$ & \\
\hline Paso 3: TMMS Subscales & 0.11 & 1.92 & & & $0.08 *$ \\
\hline Atención & & & 0.13 & 0.17 & \\
\hline Claridad & & & -0.20 & $0.04 *$ & \\
\hline Regulación & & & 0.12 & 0.29 & \\
\hline
\end{tabular}


En la primera ecuación se introdujo, la edad y sexo como predictores (covariables). En esta primera fase, el 9\% de la varianza de orientación negativa hacia los problemas fue explicada por el sexo $(\beta=-0.21 p<0.01)$ y la edad $(\beta=0.22 p<0.01)$. Las mujeres con menos edad, es decir más jóvenes, mostraban una mayor orientación negativa hacia los problemas. El resto de dimensiones del SPSI-R quedaron excluidas del primer paso de la ecuación.

En una segunda fase se introdujeron las variables del LOT-R (optimismo vs. pesimismo disposicional) como predictoras de la resolución de problemas. En este paso, el $12 \%$ de la varianza de orientación positiva hacia los problemas fue explicada por el optimismo disposicional $(\beta=0.36 \mathrm{p}<0.01)$. Los participantes que piensan que en el futuro tendrán éxito, muestran una actitud constructiva hacia la resolución de problemas y creen en su propia capacidad para resolverlos. El 24\% de la varianza de orientación negativa hacia los problemas fue explicada por el optimismo a nivel negativo $(\beta=-0.28 \mathrm{p}<0.01)$ y pesimismo $(\beta=-0.20 \mathrm{p}<0.03)$. Es decir, las personas que piensan que en el futuro obtendrán pocos éxitos y muchos fracasos, son propensos a mostrar una actitud negativa hacia la resolución de problemas, ven los problemas como irresolubles y se sienten frustrados al encontrarse con problemas en su vida. El 6\% de la varianza de estilo de estilo de impulsividad/irreflexión fue explicado por el pesimismo disposicional $(\beta=-0.34 \mathrm{p}<0.01)$. Aquellos estudiantes que piensan que en el futuro ocurrirán hechos negativos, tienden a utilizar pautas ineficaces en la resolución de problemas, es decir, se caracterizan por utilizar pocas alternativas, ser rápidos e irreflexivos en cuanto a alternativas y consecuencias, valorando poco los resultados obtenidos.

En una tercera fase se introdujeron las dimensiones de la IEP, Atención, Claridad y Reparación. En esta fase el $18 \%$ de la varianza de orientación positiva hacia los problemas fue explicada por el factor claridad emocional $(\beta=0.35 \mathrm{p}<0.01)$. Según nuestros resultados, aquellas personas que son capaces de dominar sus emociones, exhibirán una actitud constructiva y positiva hacia la resolución de problemas. Por otra parte, la orientación negativa hacia los problemas venía explicada por el factor atención emocional, aportando el $12 \%$ de la varianza. Parece ser que quienes muestran altos niveles de atención emocional tenderán a perpetuar su estado de ánimo negativo y, en consecuencia, desarrollarán una orientación negativa hacia los problemas, viéndolos como irresolubles. El 10\% de la varianza de resolución racional de problemas fue explicada por el factor claridad de sentimientos $(\beta=$ $0.24 \mathrm{p}<0.01)$. Las personas que puntúan alto en el factor claridad, se caracterizan por tener un 
buen dominio de sus emociones, por lo que cabe esperar que, sepan utilizar de forma hábil principios y técnicas de resolución de problemas. Por último, el $8 \%$ de la varianza de estilo de evitación fue explicado por el factor claridad emocional pero a nivel negativo $(\beta=-0.20$ $\mathrm{p}<0.04)$. Los sujetos con baja claridad emocional se muestran confundidos sobre sus emociones y es más probable que realicen acciones de evitación de problemas en vez de afrontarlos.

Posteriormente, con objeto de comprobar si existen diferencias de género en las dimensiones del LOT-R, TMMS y SPSI-R se llevo a cabo un análisis de varianza (ANOVA). Los resultados hallados ponen de manifiesto que, efectivamente, se encuentras diferencias estadísticamente significativas en la dimensión del TMMS, regulación emocional $(\mathrm{M}=28.30$

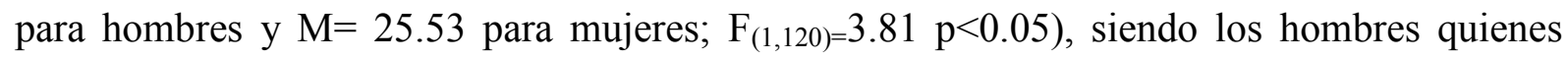
regulan mejor sus emociones comparados con las mujeres. Así mismo, también encontramos diferencias entre hombres y mujeres en la sub-escala del SPSI-R, orientación negativa hacia los problemas $\left(\mathrm{M}=14.65\right.$ para hombres y $\mathrm{M}=19.45$ para mujeres; $\left.\mathrm{F}_{(1,121)=7.79} \mathrm{p}<0.00\right)$. Este resultado muestra que las mujeres tienen una mayor orientación negativa hacia los problemas que los hombres. En el resto de variables no se encontraron diferencias estadísticamente significativas.

\section{Discusión}

En este estudio se ha constatado la validez incremental de la IEP, medida mediante el TMMS como un predictor significativo de la resolución de problemas sociales controlando las variables demográficas (edad y sexo) y el optimismo/pesimismo disposicional. Específicamente, la dimensión de claridad emocional explica parte de la varianza de orientación positiva hacia los problemas y resolución racional de problemas. Por tanto, a la luz de nuestros resultados podemos indicar que las personas con alta claridad emocional, es decir, aquellas que saben discernir y comprender sus estados emocionales en el momento que se enfrentan a un estímulo estresante, como podría ser la percepción de un determinado problema, obtendrán beneficios de ésta habilidad. De este modo estos individuos valorarán el problema como un desafío, creerán en su propia capacidad para resolverlo y se comprometerán a enfrentarlo en vez de poner en marcha estrategias de evitación. Así mismo, cabe esperar que se trate de personas cuidadosas y sistemáticas a la hora de reunir aquella información que les permita identificar las posibles demandas y obstáculos, en el 
establecimiento de sus metas. Estos resultados se muestran en consonancia con los hallados por Gohm y Clore (2002) y Gohm, Baumann y Sniezek (2001). Según los autores, las personas que tienen mayor facilidad para comprender el origen de sus emociones en situaciones estresantes, son más habilidosos para tratar problemas sociales e invierten menos tiempo en atender a sus reacciones emocionales. Es decir, utilizan menos recursos cognitivos cuando evalúan las alternativas de acción, lo que les permite mantener sus pensamientos en otras tareas o utilizar estrategias de afrontamiento más adaptativas. Por otro lado, una claridad emocional baja predice parte de la varianza de estilo de impulsividad, es decir, cuando las personas están confundidas respecto a sus emociones es más probable que utilicen un estilo de solución de problemas caracterizado por aplazar la solución, mostrar pasividad y/o dependencia. Tal y como apuntan Extremera y Fernández-Berrocal (2005b), la probabilidad de que en personas con baja claridad emocional, se produzcan reacciones imprevisibles, y a menudo problemáticas ante situaciones emocionales, es mayor que en el caso de puntuar alto en dicha dimensión.

En cuanto al optimismo vs. pesimismo disposicional, nuestros resultados indican que el optimismo predice parte de la varianza de orientación positiva hacia los problemas, es decir, que aquellas personas que puntúan alto en optimismo, manifiestan una predisposición optimista hacia los problemas, evaluándolos a partir de la idea de que van a poder solucionarlos. En esta misma dirección apuntan los resultados obtenidos por Vera-Villarroel y Guerrero (2003), señalando que las personas optimistas tienden a enfrentarse de forma positiva hacia los problemas, lo cual es uno de los primeros requisitos para poder solucionarlos de manera adecuada. Así mismo, el pesimismo disposicional explica parte de la varianza de orientación negativa hacia los problemas, el estilo de impulsividad y el estilo de evitación. Como era de esperar, las personas que creen que en el futuro les ocurrirán hechos desfavorables o negativos, utilizaran una orientación negativa hacia los problemas, lo que en términos conductuales se va a traducir en la puesta en marcha de un estilo impulsivo y de evitación del problema.

En cuanto a las diferencias de género solamente encontramos diferencias significativas en una sola escala del TMMS, la regulación emocional. Según los resultados hallados en el presente estudio, los hombres mostrarían mayor capacidad de interrumpir sus estados emocionales negativos y prolongar sus estados los positivos, resultado en línea a los obtenidos en estudios previos. Es decir, las mujeres regulan peor sus estados emocionales que los 
hombres (Fernández-Berrocal y Extremera, 2003, Thayer, Rossy, Ruiz-Padial y Johnsen, 2003). Por otra parte, hemos encontrado diferencias significativas en la sub-escala del SPSI$\mathrm{R}$, orientación negativa hacia el problema. Las mujeres tienden en mayor medida que los hombres, a percibir el problema como una amenaza, esperando que no tenga solución o bien dudando de su habilidad para resolverlo, lo que les puede llevar a experimentar sentimientos de frustración cuando tienen problemas, resultados similares a los mostrados por Morera et al.(2006). Por otra parte, las mujeres del estudio muestran poca capacidad de interrumpir los estados emocionales negativos lo que significa que tendrán mayor probabilidad de exhibir una orientación negativa hacia los problemas.

Si bien nuestro estudio nos aporta una interesante evidencia preliminar sobre la validez incremental del TMMS y el optimismo/pesimismo disposicional, ante la resolución de problemas sociales, los resultados obtenidos en la presente investigación deben ser interpretados con cierta cautela debido a las limitaciones propias de este estudio. En primer lugar, una de las posibles limitaciones se debe a los bajos índices de consistencia interna obtenidos en la escala LOT-R (0,62 optimismo y 0,48 pesimismo). El motivo por el que quizás, podrían justificarse estos índices, podría encontrarse en que en este trabajo se han aplicado los ítems relativos al optimismo y los relativos al pesimismo, como dos escalas separadas, lo que ha podido influenciar la respuesta dada por los participantes a la misma (Chang, Sanna y Yang, 2003; Extremera et al. 2007). En segundo lugar, respecto a la medida de la IEP, creemos necesario utilizar pruebas objetivas en futuras líneas de investigación. En este sentido, deberían utilizarse medidas de habilidad tales como el MSCEIT, basado también en el modelo propuesto por Mayer y Salovey (1997), de forma que, además de medir la IEP de manera objetiva, podamos evaluar el estilo con el que las personas resuelven determinados problemas emocionales comparando sus respuestas con criterios de puntuación determinados y objetivos. Así mismo, sería importante el diseño de entrenamientos sobre habilidades de inteligencia emocional, tal y como proponen Ciarrochi y Mayer (2007), con el fin de comprobar su impacto en la resolución de problemas sociales que muestran los alumnos de trabajo social. 


\section{Referencias}

Augusto-Landa, J. M., López-Zafra, E., Martínez, R., y Pulido, M. (2006). Perceived emotional intelligence and life satisfaction among university teachers. Psicothema, 18 , $152-157$.

Avia, M. D. y Vázquez, C. (1999). Optimismo Inteligente. Psicología de las emociones positivas. Madrid: Alianza Editorial.

Berrios-Martos, M.P., Augusto-Landa, J.M., y Aguilar-Luzón, M.C. (2006). Inteligencia emocional percibida y satisfacción laboral en contextos hospitalarios. Un estudio exploratorio con profesionales de enfermería. Index de Enfermería, 54: 30-34.

Calero, M. D., Luna, M. J., Vera-Villarroel, P., y González Trujillo, M. C. (2001). Un estudio de validez del inventario de solución de problemas sociales (Social Problem-Solving Inventory-R, SPSI-R). Psicología Conductual, 9 (2), 373-387.

Carver, C., y Scheier, M. (2001). Optimism, pessimism, and self-regulation. In E. Chang (Ed.). Optimism and pessimism. Implications for theory, research and practice (pp. 31-51). Washington, DC: American Psychological Association.

Chang, E. C. (1998). Cultural differences, perfectionism, and suicidal risk in a college population: Does social problem solving still matter? Cognitive Therapy and Research, 22, 237-254.

Chang, E. C. (2001). Introduction: optimism and pessimism and moving beyond the most fundamental question. En E. C. Chang (Ed.). Optimism and pessimism. Implications for theory, research and practice (pp. 4-12). Washington, DC: American Psychological Associaton.

Chang, E. C., Sanna, L. J., y Yang, K. M. (2003). Optimism, pessimism, affectivity, and psychological adjustment in US and Korea: a test of mediation model. Personality and Individual Differences, 34, 1195-1208.

Chico, E. (2002). Optimismo disposicional como predictor de estrategias de afrontamiento. Psicothema 14 (3), 544-550.

Ciarrochi, J., y Mayer, J. D. (2007). Applying emocional intelligence: A practitioners' guide. New York and Hove. Psychology Press.

D'Zurilla, T. J., y Nezu, A. M. (1990). Development and preliminary evaluation of the social problema-solving inventory. Psychological Assessment, 2 (2), 156-163.

D'Zurilla, T. J., y Nezu, A. M. (1999). Problem solving therapy. New York: Springer Publishing Company. 
D'Zurilla, T. J., Maydeu-Olivares, A., y Kant, G. L. (1998). Age and gender differences in social problema-solving ability. Personality and Individual Differences, 25, 241-252.

D’Zurilla, T. J., Nezu, A. M., y Maydeu-Olivares, A. (1998). Manual for the social problemasolving inventory revised (SPSI-R), North-Tonawanda, NY: Multi-Health Systems, Inc.

D`Zurilla, T. J., Nezu, A. M., y Maydeu-Olivares, A. (2004). Social problem solving: Theory and assessment. In E. C. Chang, T. J. D’Zurilla, y L. J. Sanna (Eds.). Social problem solving: Theory, research, and training. Washington, D. C.: American Psychological Association.

Esquivias, M.T., González, A. y Muria, I. (2003). Solución de Problemas: Estudio evaluativo de tres enfoques pedagógicos en las escuelas mexicanas. Electronic Journal of Research in Educational Psychology, 1(2), 79-96.

Extremera, N., Durán, A., y Rey, L. (2007). Perceived emotional intelligence and dispositional optimism-pessimism: Analyzing their role in predicting psychological adjustment among adolescents. Personality and Individual Differences, 42, 10691079 .

Extremera, N., y Fernández-Berrocal, P. (2005a). Perceived emotional intelligence and life satisfaction: Predictive and Incremental validity using the Trait Meta-mood Scale. Personality and Individual Differences, 39, 937-948.

Extremera, N., y Fernández-Berrocal, P. (2005b). Inteligencia emocional percibida y diferencias individuales en el meta-conocimiento de los estados emocionales: Una revisión de los estudios con el TMMS. Ansiedad y Estrés, 11 (2-3), 101-122.

Fernández-Berrocal, P., y Extremera, N. (2003). ¿En qué piensan las mujeres para tener un peor ajuste emocional? Encuentros en Psicología Social, 1 (5), 255-259.

Fernández-Berrocal, P., Extremera, N., y Ramos, N. (2004). Validity and reliability of the Spanish modified version of the Trait Meta-Mood Scale. Psychological Reports, 94, 751755.

Ferrando, P. J., Chico, E., y Tous, J. M. (2002). Propiedades psicométricas del test de optimismo Life Orientation Test. Psicothema, 14 (3), 673-680.

Gillham, J. E., Shatté, A. J., Reivich, K. J., y Seligman, M. E. P. (2001). Optimism, pessimism and explanatory style. En E. C. Chang (Ed.). Optimism and pessimisms. Implications for theory, research and practice (pp. 53-75). Washington, DC: American Psychological Associaton 
Gohm, C. L., Baumann, M. R., y Sniezek, J. A. (2001). Personality in extreme situations: Thinking (or not) under acute stress. Journal of Research in Personality, 35, 288-399.

Gohm, C. L., y Clore, G. L. (2002). Four latent traits of emotional experience and their involvement in attributional style, coping and well-being. Cognition and Emotion, 16, 495-518.

Kant, G. L., D'Zurilla, T. J., y Maydeu-Olivares, A. (1997). Social problema-solving as a mediator of stress-related depression and anxiety in middle-aged and elderly community residents. Cognitive Therapy and Research, 21 (1), 73-96.

Martínez-Correa, A., Reyes del Paso, G., García-León, A., y González-Jareño, M. I. (2006). Optimismo/pesimismo disposicional y estrategias de afrontamiento del estrés. Psicothema, 18 (1), 66-72.

Maydeu-Olivares, A., y D'Zurilla, T. J. (1996). A factor-analytic study of the social problema-solving inventory: An integration of theory and data. Cognitive Therapy and Research, 20 (2), 115-133.

Maydeu-Olivares, A., D'Zurilla, T. J., y Kant, G. L. (1994). Age and differences in social problem solving in college student, middle-aged and elderly adults. Paper at the $66^{\text {th }}$ Annual Meeting of the Midwesatern Psychological Association. Chicago (IL).

Mayer, J. y Salovey, P. (1997). What is emocional intelligence? In P. Salovey y D. Sluyter (Eds.), Emotional development and emotional intelligence: implications for Educators. New York: Basic Books.

Morera, O. F., Madey-Olivares, A., Nygren, T. E., White, R. J., Fernández, N. P., y Skewes, M. C. (2006). Social problem solving predicts decision making styles among US Hispanics. Personality and Individual Differences, 41, 307-317.

Nezu, A. M. y 'Zurilla, T. J. (1989). Social problema solving and negative affective conditions. In P. C. Kendall y D. Watson (Dir.). Anxiety and depression: distinctive and overlapping features (pp. 285-315). New York: Academic Press.

Otero, J. M., Luengo, A., Romero, E., Gómez, J. A., y Castro, C. (1998). Psicología de la Personalidad. Manual de prácticas. Barcelona: Ariel Prácticum.

Remor, E., Amorós, M., y Carboles, J. A. (2006). El optimismo y la experiencia de ira en relación con el malestar físico. Anales de Psicología, 22, 37-44.

Salovey, P. y Mayer, J. D. (1990). Emotional intelligence. Imagination, Cognition, And Personality, 9, 185-211.

Salovey, P., Mayer, J, D., Goldman, S., Turvey, C., y Palfai, T. (1995). Emotional attention, clarity, and repair: Exploring emotional intelligence using the Trait Meta-Mood Scale. 
En J. W. Pennebaker (Ed,). Emotion, disclosure and health (pp. 125-154). Washington, D. C.: American Psychological Association.

Salovey, P., Woolery, A., Stroud, L., y Epel, E. (2002). Perceived emotional intelligence, stress reactivity and symptom reports: Furthers explorations using the Traid MetaMood Scale. Psychology and Health, 17, 611-627.

Sheier, M., Carver, C., y Bridges, M. (1994). Distinguishing optimism from neuroticism (and trait anxiety, self-mastery, and self-esteem): A re-evaluation of the Life Orientation Test. Journal of Personality and Social Psichology, 67, 1063-1078.

Thayer, J. F., Rossy, L. A., Ruiz-Padial, E., y Johnsen, B. H. (2003). Gender differences in the relationship betweem emotional regulation and depressive symptoms. Cognitive Therapy and Research, 27, 349-364.

Thompson, B. L., Waltz, J., Croyle, K., y Pepper, A. C. (2007). Trait meta-mood and affect as predictors of somatic symptoms and life satisfaction. Personality and Individual Differences, 43, 1786-1795.

Vera-Villarroel, P. E. y Guerrero, A. (2003). Diferencias en habilidades de resolución de problemas sociales en sujetos optimistas y pesimistas. Universitas Psicológica, 2 (1), 21-26. 
José María Augusto Landa et al.

\section{[Página en blanco por razones de paginación]}

